\title{
Relação entre bem-estar espiritual, características sociodemográficas e consumo de álcool e outras drogas por estudantes
}

\author{
Relationship between spiritual well-being, sociodemographic \\ characteristics and use of alcohol and other drugs by students \\ Roberta de Paiva Silva', Priscila de Souza', Denismar Alves Nogueira', Denis da Silva Moreira', \\ Erika de Cássia Lopes Chaves?
}

\section{RESUMO}

Objetivos: O estudo objetivou avaliar o consumo de álcool e de outras drogas por estudantes de Enfermagem e investigar a relação entre esse consumo e o bem-estar espiritual e as características sociodemográficas/religiosas dos estudantes. Métodos: Trata-se de um estudo epidemiológico, observacional e seccional, realizado com 100 graduandos do curso de Enfermagem de uma universidade do sul de Minas Gerais. A coleta de dados foi realizada no segundo semestre de 2011 e utilizou um questionário sociodemográfico, a Escala de Bem- estar Espiritual e o Teste de Triagem do Envolvimento com Álcool, Tabaco e outras Substâncias. O tratamento estatístico dos dados foi realizado utilizando-se a análise univariada, por meio do teste qui-quadrado e análise múltipla, por meio da regressão logística dicotômica. Resultados: $\mathrm{O}$ álcool demonstrou ser a substância mais utilizada pelos estudantes (84\%), o que, por sua vez, apresentou relação significativa com a espiritualidade deles, visto que possuir bem-estar espiritual negativo (OR: 3,325; IC95\%: 1,059-10,441) e não ter prática religiosa frequente (OR: 3,392; IC95\%: 1,064-10,811) aumenta as chances de consumo abusivo dessa

\section{Palavras-chave}

Enfermagem, transtornos causados pelo uso de substâncias, espiritualidade. substância. Conclusão: Iniciativas preventivas ao consumo de drogas psicoativas, vinculadas à prática de atividades espirituais, podem ser utilizadas como estratégias para a promoção de hábitos saudáveis e para a manutenção da saúde e também como recurso na formação profissional de estudantes de Enfermagem.

\section{ABSTRACT}

Objectives: This study aimed to evaluate the use of alcohol and other drugs by nursing students and to investigate the relationship between this consumption, and the spiritual well-being and sociodemographic characteristics/religious students. Methods: This was an exploratory study of type epidemiological and cross-sectional, conducted with 100 graduating students of nursing at a university in southern Minas Gerais. The data collection was conducted in the second half of 2011 using a sociodemographic questionnaire, the Scale

1 Universidade Federal de Alfenas (Unifal-MG), Programa de Pós-Graduação em Enfermagem.

Endereço para correspondência: Erika de Cássia Lopes Chaves

Programa de Pós-Graduação em Enfermagem, Universidade Federal de Alfenas

Rua Gabriel Monteiro da Silva, 700, Centro - 37130-000 - Alfenas, MG, Brasil

Telefone: (35) 3299-1380

E-mail: echaves@unifal-mg.edu.br 


\section{Keywords}

Nursing, substance-related disorders, spirituality. and Spiritual Well-being, Screening Test of Involvement with Alcohol, Tobacco and other Substances. The statistical treatment of the data was performed using univariated analysis, through chi square test and multiple analyses, by logistic dichotomic regression. Results: Alcohol proved to be the most commonly used substance among students (84\%), which in turn, showed a significant relationship with the spirituality of the same, seen that owning spiritual welfare negative (OR: 3,325; IC95\%: 1,059-10,441) and having no religious frequent practice (OR: 3,392; IC95\%: 1,064-10,811) increase the chances of abuse of this substance. Conclusion: Preventive initiatives to the consumption of psychoactive drugs linked to the practice of spiritual activities can be used as strategies to promote healthy habits and health maintenance, and also as a resource in the training of nursing students.

\section{INTRODUÇÃO}

Os danos decorrentes do uso do álcool e outras drogas são amplos e interferem em diversos aspectos da vida do indivíduo, resultando em comportamento de risco à saúde que afeta o desenvolvimento físico, mental e social; esse comportamento se inicia, comumente, na adolescência e se estende por toda a juventude ${ }^{1,2}$.

Em 2010, foi divulgado o I Levantamento Nacional sobre Uso de Álcool, Tabaco e outras Drogas entre 12.711 Estudantes Universitários, realizado pela Secretaria Nacional de Políticas sobre Drogas (Senad), o qual revelou que quase a metade desses universitários (49\%) já usou drogas ilícitas. O estudo notificou, ainda, que $22 \%$ dos estudantes estavam sob o risco de desenvolver dependência de álcool e 3\% já apresentavam o padrão de dependência ${ }^{3}$.

Para estudantes universitários, o consumo de álcool e outras drogas pode ocasionar, além das diversas consequências pessoais e sociais, importante prejuízo ao processo de profissionalização, como mau desempenho acadêmico, por causa das faltas ou dos atrasos constantes nas atividades escolares, do baixo rendimento durante as aulas e nos exames das disciplinas e dos inúmeros problemas de saúde, que repercutirão durante toda a formação acadêmica .

Entre estudantes de Enfermagem, as implicações remetem, ainda, ao seu papel como profissionais na área de saúde, pois deverão ser capazes de desenvolver ações profiláticas no campo social e de saúde pública; nesse sentido, esses futuros profissionais servirão como modelo para seus pacientes, o que torna maior sua responsabilidade em evitar o uso de substâncias psicoativas 5 .

Outro importante fator relacionado ao graduando de Enfermagem é o fato de lidar com o ser humano no confronto direto com o sofrimento, com a dor e com a morte e ao mesmo tempo a deficiência de um processo educativo que prepare os alunos para enfrentar esses problemas ${ }^{6}$. Por conseguinte, é um público cuja atenção para a magnitude do consumo de álcool e outras drogas e de suas consequências requer o envolvimento de pesquisadores e de educadores dessa área de atuação.

A inclusão da dimensão espiritual como um fator de proteção para o uso de álcool e outras drogas é, ao mes- mo tempo, inovador e relevante. Uma revisão de literatura, realizada em 2007, demonstrou que indivíduos que dão importância à espiritualidade apresentam menores índices de consumo dessas substâncias 7 . Ainda, pesquisadores ${ }^{8}$ têm confirmado que, ao envolver-se com padrões de religiosidade e espiritualidade, o jovem adere a um conjunto de valores, de símbolos, de comportamentos e de práticas sociais, que inclui, entre outras coisas, a recusa ao uso de álcool e outras drogas. Nesse sentido, estratégias que envolvam a espiritualidade poderiam contribuir de forma significativa para o enfrentamento das dificuldades encontradas durante a vida universitária e, consequentemente, poderiam atuar como proteção para o comportamento de risco relacionado ao uso de drogas.

Vale ressaltar que espiritualidade e religiosidade são dois construtos distintos, uma vez que espiritualidade consiste em todas as crenças e atividades pelas quais a pessoa tenta relacionar sua vida a Deus, ao Ser Divino ou mesmo a outra conexão de realidade transcendente 9 . Já religião é descrita como um sistema de doutrina específica partilhada por um grupo ${ }^{10}$ e religiosidade é a manifestação exterior das crenças e cultos ${ }^{11}$.

Tendo em vista que a questão da espiritualidade é muito vasta e sua mensuração é bastante complexa, tem-se no bem-estar espiritual, ou seja, na percepção subjetiva de bem-estar do sujeito em relação à sua crença, um de seus aspectos passíveis de avaliação ${ }^{12}$.

O bem-estar espiritual está relacionado à abertura da pessoa para a dimensão espiritual associada a outras dimensões da vida, maximizando seu potencial de crescimento e autoatualização ${ }^{13}$. Portanto, a definição de "bem-estar espiritual" compreende uma sensação de bem-estar que é vivenciada quando se encontra um propósito que explique um comprometimento com algo na vida, e esse propósito envolve um "significado maior" para o indivíduo ${ }^{14}$.

Estudo $^{12}$ que examinou a influência do bem-estar espiritual na saúde mental de estudantes universitários concluiu que ele atua como fator protetor para transtornos psiquiátricos menores, considerando-se que a espiritualidade é um recurso para a promoção de saúde mental. Diante disso, o bem-estar espiritual pode ser uma importante variável no 
estudo de fatores que possam contribuir para a prevenção do uso de drogas ou, ainda, dos danos associados ao seu consumo.

Por fim, é importante destacar que uma das dificuldades encontradas para enfrentar os problemas relacionados ao uso de drogas é a falta de informações confiáveis sobre o assunto, pois, muitas vezes, os dados são divulgados fora de um contexto, sem fundamento na realidade ou de forma distorcida, contribuindo para uma visão preconceituosa sobre o consumo de álcool e drogas ${ }^{3}$. Assim, este estudo objetivou avaliar o consumo de álcool e outras drogas por estudantes de enfermagem e investigar a relação entre esse consumo e o bem-estar espiritual e as características sociodemográficas/religiosas dos estudantes.

\section{MÉTODOS}

Trata-se de um estudo epidemiológico, observacional e seccional, realizado em uma universidade do sul de Minas Gerais. A população eleita foi de estudantes matriculados no curso de graduação em Enfermagem, que, no segundo semestre de 2011, totalizaram 157 alunos, divididos nos segundo, quarto, sexto e oitavo período. Desses, participaram do estudo 100 alunos, compondo a amostragem aleatória simples em que foi respeitado o critério de inclusão: idade acima de 18 anos. Foram excluídos os indivíduos que estavam afastados das atividades didáticas por atestados médicos ou que não compareceram às aulas por três dias consecutivos.

Os instrumentos utilizados foram o questionário de caracterização dos sujeitos de estudo, a Escala de Bem-estar Espiritual (EBE) ${ }^{13}$ e o Teste de Triagem do Envolvimento com Álcool, Tabaco e outras Substâncias (ASSIST) ${ }^{15}$.

O questionário de caracterização dos sujeitos foi desenvolvido com base na literatura de referência ${ }^{1,16}$ e teve a finalidade de investigar, além dos aspectos sociodemográficos (sexo, idade, estado civil e renda familiar mensal), também aqueles relacionados à religiosidade dos sujeitos, a saber, prática religiosa; tempo de prática religiosa; importância da religião; prática religiosa familiar e importância da religiosidade na formação do profissional de saúde. Esse instrumento foi submetido a um processo de refinamento de conteúdo e de aparência, que contou com a avaliação de um grupo de cinco juízes, peritos na temática investigada.

Sob a coordenação da Organização Mundial de Saúde (OMS), pesquisadores de vários países desenvolveram um instrumento para detecção do uso de álcool, tabaco e outras substâncias psicoativas, denominado ASSIST. Esse instrumento foi traduzido para várias línguas, inclusive para o português falado no Brasil, já tendo sido testado quanto à sua confiabilidade e factibilidade, quando aplicado por Henrique et al. em 2004'15. O estudo da confiabilidade teste-reteste do ASSIST foi realizado com 236 indivíduos, em diferentes locais do mundo ${ }^{17}$, e foi observada uma boa confiabilidade com kappa entre 0,58 e 0,90 para as principais questões.

O ASSIST é um questionário estruturado contendo oito questões sobre o uso de nove classes de substâncias psicoativas: tabaco, álcool, maconha, cocaína, estimulantes, sedativos, inalantes, alucinógenos e opiáceos. Vale ressaltar que o instrumento visa ao rastreamento do uso dessas substâncias para a identificação de situações de risco, o que exclui o uso por indicação médica, conforme explícito nas instruções para preenchimento dele.

As questões do ASSIST englobam a frequência de uso de substâncias psicoativas, na vida e nos últimos três meses; os problemas decorrentes dele; as preocupações, por parte de pessoas próximas ao usuário, acerca do consumo; os prejuízos na execução de tarefas esperadas; as tentativas malsucedidas de cessar ou de reduzir seu consumo; o forte desejo ou a urgência em consumir drogas e o uso por via injetável. Cada resposta corresponde a um escore, que varia de 0 a 4 , e a soma total pode variar de 0 a 20. Considera-se a faixa de escore de 0 a 3 como indicativa de uso ocasional; de 4 a 15 como indicativa de abuso e maior que 16 como sugestiva de dependência ${ }^{15}$.

A EBE foi desenvolvida por Paloutzian e Elisson, em $1982^{18}$, e adaptada e validada para população brasileira por Marques et al., em 2003, cuja consistência interna foi de 0,92, revelando alta fidedignidade ${ }^{13}$. Esse instrumento é constituído por 20 itens; 10 itens são destinados à avaliação do bem-estar religioso e os demais à mensuração do bem-estar existencial ${ }^{12}$. Os itens são respondidos em uma escala de seis pontos, do tipo Likert, que varia de "concordo fortemente" a "discordo fortemente". Os pontos de corte para o escore geral da EBE são os intervalos de 20 a 40, 41 a 99 e 100 a 120, para baixo, moderado e alto bem-estar espiritual, respectivamente. Nas duas subescalas, de bem-estar religioso e de bem-estar existencial, os intervalos são 10 a 20, 21 a 49 e 50 a 60 pontos, para baixo, moderado e alto, respectivamente ${ }^{18}$. Na análise deste estudo, os resultados da EBE foram denominados positivos para escore alto, e negativo para o moderado e o baixo ${ }^{12,19}$

A coleta de dados foi realizada no segundo semestre de 2011. Antes da realização da coleta, foi apresentado o Termo de Consentimento Livre e Esclarecido aos participantes do estudo e, mediante a sua concordância em participar da pesquisa, foram apresentados os instrumentos de coleta de dados.

Os instrumentos autoaplicáveis foram colocados em envelope e entregues aos alunos no início das atividades didáticas. Ao final do dia, posteriormente ao preenchimento, os instrumentos foram recolhidos em envelope lacrado. Essa forma de coleta foi utilizada para possibilitar ao aluno o preenchimento no momento que considerasse mais adequado e para garantir seu anonimato. 
A análise dos dados, realizada pelo programa estatístico Statistical Package for the Social Sciences (SPSS), versão 17,0, foi precedida da elaboração de um banco de dados que foi submetido à dupla digitação para validação dele. A estatística descritiva foi utilizada para descrever e resumir os dados obtidos. Foi empregada a análise univariada, por meio do teste qui-quadrado, para investigar a relação entre o consumo de substâncias psicoativas e o bem-estar espiritual e as características sociodemográficas/religiosas dos estudantes. A análise múltipla foi utilizada para investigar a influência do bem-estar espiritual e da religiosidade sobre o uso de drogas; ela foi baseada em odds ratio, estimado pelo modelo de regressão logística dicotômica. O método de seleção das variáveis utilizado foi Backward, com nível de significância de 0,10; portanto, inicialmente, todas as variáveis independentes foram incluídas na análise e, posteriormente, passo a passo, as possíveis combinações de variáveis foram feitas até se chegar àquela que melhor discriminou os níveis da variável dependente.

Para assegurar os direitos dos participantes em cumprir os aspectos contidos na Resolução no 196/96, do Ministério da Saúde, que trata das diretrizes e normas preconizadas em pesquisa envolvendo seres humanos, o estudo foi submetido a um Comitê de Ética em Pesquisa e aprovado sob o Protocolo no 120/2010.

\section{RESULTADOS}

Dos 100 acadêmicos que participaram deste estudo, 87,0\% foram do sexo feminino, sendo a proporção de sete mulheres para cada homem. Os acadêmicos investigados se apresentaram jovens (21 anos); solteiros (98,0\%); residentes em república $(61,0 \%)$ e com nível socioeconômico (renda familiar mensal) entre quatro e cinco salários (46,0\%).

As características religiosas da população e os escores de bem estar-espiritual estão representados na tabela 1. É importante destacar que, no que se refere ao bem-estar espiritual e existencial, verificou-se que as frequências de escores positivos e negativos foram semelhantes, enquanto a frequência de bem-estar religioso positivo foi consideravelmente maior. As análises de consistência interna dos itens da Escalas de Bem-estar Espiritual demonstraram alta fidedignidade, com alfa de Cronbach de 0,889.

As frequências das respostas positivas ao ASSIST estão evidenciadas na tabela 2, na qual é demonstrado que, entre os estudantes que experimentaram maconha, anfetaminas, inalantes e opiáceos, nenhum deles fez uso nos últimos três meses que antecederam à pesquisa; no entanto, em relação às substâncias lícitas (álcool e tabaco), mais da metade dos estudantes refere uso nos últimos três meses e, ainda, mais de $80 \%$ deles fizeram o uso experimental de álcool na vida de forma geral.
Conforme o instrumento desenvolvido pela OMS, o ASSIST, o uso das substâncias psicoativas é caracterizado em ocasional, sugestivo de abuso e sugestivo de dependência; dessa forma, o perfil de consumo de álcool e outras drogas é apresentado na tabela 3. Entre os acadêmicos que consomem álcool, $74 \%$ fazem uso ocasional e $26 \%$ fazem uso indicativo de abuso. No que se refere ao tabaco, 58\% consomem ocasionalmente, 39\% fazem uso indicativo de abuso e $3 \%$ apresentam provável dependência. Entre os que utilizam hipnóticos/sedativos, 50\% fazem uso ocasional e os demais fazem uso sugestivo de abuso. As demais substâncias investigadas foram identificadas somente como de uso ocasional.

Tabela 1. Distribuição dos acadêmicos de Enfermagem quanto às características religiosas/espirituais e à Escala de Bem-Estar Espiritual e subescalas - Minas Gerais, 2012

\begin{tabular}{|c|c|}
\hline Caracteristicas & $\%(n=100)$ \\
\hline \multicolumn{2}{|l|}{ Religião } \\
\hline Católica & 74 \\
\hline Evangélica & 13 \\
\hline Espírita & 5 \\
\hline Sem religião & 8 \\
\hline \multicolumn{2}{|c|}{ Tempo em que pratica a religião } \\
\hline Um a cinco anos & 6 \\
\hline Mais de cinco anos & 94 \\
\hline \multicolumn{2}{|l|}{ Prática religiosa } \\
\hline$\geq$ uma vez por mês & 72 \\
\hline$<$ uma vez por mês & 28 \\
\hline \multicolumn{2}{|c|}{ Importância da religião } \\
\hline Importante & 92 \\
\hline Não importante & 8 \\
\hline \multicolumn{2}{|c|}{$\begin{array}{l}\text { A influência religiosa dos pais na infância foi importante } \\
\text { na determinação da religião do aluno }\end{array}$} \\
\hline $\operatorname{sim}$ & 83 \\
\hline Não & 17 \\
\hline \multicolumn{2}{|c|}{ Espiritualidade é importante na formação do profissional de saúde } \\
\hline Importante & 90 \\
\hline Não é importante & 10 \\
\hline \multicolumn{2}{|c|}{ Escala de Bem-Estar Espiritual } \\
\hline \multicolumn{2}{|l|}{ Bem-estar espiritual } \\
\hline Positivo* & 56 \\
\hline Negativo* & 44 \\
\hline \multicolumn{2}{|l|}{ Bem-estar existencial } \\
\hline Positivo & 50 \\
\hline Negativo & 50 \\
\hline \multicolumn{2}{|l|}{ Bem-estar religioso } \\
\hline Positivo & 77 \\
\hline Negativo & 23 \\
\hline
\end{tabular}


Tabela 2. Frequência dos estudantes de Enfermagem que pontuaram em cada questão do ASSIST, segundo a substância - Minas Gerais, 2012

\begin{tabular}{|c|c|c|c|c|c|c|c|}
\hline \multirow{2}{*}{ Substâncias } & \multicolumn{7}{|c|}{ Frequência de respostas positivas } \\
\hline & $\begin{array}{l}\text { Uso } \\
\text { na vida }\end{array}$ & $\begin{array}{l}\text { Uso nos três últimos } \\
\text { meses }\end{array}$ & $\begin{array}{l}\text { Desejo ou urgência em } \\
\text { consumir }\end{array}$ & Problemas associados & $\begin{array}{l}\text { Negligência de } \\
\text { atividades }\end{array}$ & Preocupação de outros & Tentativa de reduzir \\
\hline Tabaco & 36 & 21 & 11 & 2 & 1 & 6 & 9 \\
\hline Álcool & 84 & 77 & 36 & 13 & 9 & 17 & 7 \\
\hline Maconha & 12 & 0 & 0 & 0 & 0 & 0 & 0 \\
\hline Anfetaminas & 4 & 0 & 0 & 0 & 0 & 0 & 0 \\
\hline Inalantes & 5 & 0 & 0 & 0 & 0 & 0 & 0 \\
\hline Sedativos & 4 & 2 & 2 & 0 & 0 & 1 & 2 \\
\hline Opiáceos & 1 & 1 & 0 & 0 & 0 & 0 & 0 \\
\hline
\end{tabular}

Tabela 3. Frequência do uso de substâncias por estudantes de Enfermagem segundo os escores totais do ASSIST para uso ocasional, sugestivo de abuso e sugestivo de dependência - Minas Gerais, 2012

\begin{tabular}{lcccc}
\hline Tipo de droga & Uso ocasional & Uso sugestivo de abuso & Uso sugestivo de dependência & Total \\
\hline Tabaco & 21 & 14 & 1 & 36 \\
Álcool & 62 & 22 & 0 & 84 \\
Maconha & 12 & 0 & 0 & 0 \\
Cocaína & 0 & 0 & 0 & 4 \\
Anfetaminas & 4 & 0 & 0 & 5 \\
Inalantes & 5 & 0 & 0 & 4 \\
Hipnóticos/sedativos & 2 & 2 & 0 & 0 \\
Alucinógenos & 0 & 0 & 0 & 1 \\
Opioides & 1 & 0 & 0 \\
\hline
\end{tabular}

Ao relacionar o padrão de uso de substâncias psicoativas com as características sociodemográficas, religiosas e de bem-estar espiritual dos sujeitos investigados, observou-se, por meio da análise univariada dos dados, que não houve diferenças estatisticamente significativas. Entretanto, vale lembrar que apenas o álcool e o tabaco foram as substâncias mais utilizadas, em termos de frequência absoluta, pelos estudantes (Tabela 3).

Assim, ao realizar a regressão logística dicotômica múltipla, elegeu-se como variáveis dependentes apenas o uso de álcool e tabaco, uma vez que, no estudo, apenas essas drogas apresentaram uma frequência válida de uso sugestivo de abuso e não só padrão de uso ocasional, como observado para as demais substâncias. Entretanto, apenas o uso de álcool obteve associações significantes, o que resultou num modelo final ajustado.

Portanto, os resultados da regressão logística dicotômica múltipla evidenciaram que possuir bem-estar espiritual negativo aumenta em 3,3 vezes a chance de fazer uso abusivo de álcool $(p=0,040)$ e que os alunos que não têm prática religiosa frequente apresentam 3,4 vezes mais chances de fazer uso abusivo dessa substância $(p=0,039)$ (Tabela 4). 0 ajuste do modelo de regressão apresentou um coeficiente de determinação de $R^{2}=17 \%$.
Tabela 4. Estimativa dos parâmetros do modelo de regressão logística dicotômica e os fatores associados ao uso abusivo de álcool por estudantes de Enfermagem - Minas Gerais, 2012

\begin{tabular}{lccccc}
\hline Variáveis & Parâmetros & Erro-padrão & OR & IC95\% & valorp \\
\hline Prática religiosa & 1,221 & 0,591 & 3,392 & $1,064-10,811$ & $0,039^{*}$ \\
Bem-estar espiritual & 1,201 & 0,584 & 3,325 & $1,059-10,441$ & $0,040^{*}$ \\
\hline
\end{tabular}

IC95\%: intervalo de confiança de 95\%; OR: odds ratio; *: $P<0,05$.

\section{DISCUSSÃO}

As taxas de consumo de álcool e outras drogas por universitários, especialmente as de substâncias lícitas, que foram identificadas como mais elevadas neste estudo, corroboraram outros achados da literatura ${ }^{20-22}$ e vêm representando um tema de grande apreensão não só aos pais de jovens, mas também aos educadores, aos profissionais de saúde e à sociedade em geral. Particularmente, o uso do tabaco e do álcool é uma questão preocupante por diversas justificativas, entre elas, o não reconhecimento pelos estudantes dos riscos que o consumo acarreta, pelo fato de serem consideradas de uso comum e de serem aceitas socialmente.

Outras pesquisas também têm encontrado baixo consumo de substâncias ilícitas em comparação com o uso de substâncias lícitas entre estudantes de Enfermagem²3, o 
que pode estar relacionado às características do curso, que é constituído predominantemente por mulheres, além de, muitas vezes, de profissionais que já atuam na área. Ainda, em estudo conduzido no México ${ }^{24} \mathrm{Com}$ universitários, foi observada menor prevalência de consumo de drogas pelo sexo feminino, especificando que essa característica se estende à população em geral e tem sido justificado que as mulheres têm mais restrições para realizar determinados comportamentos de risco relacionados a substâncias ilegais, voltando o consumo para aquelas de fácil acesso ${ }^{25}$.

Independentemente do perfil de consumo, que pode variar em relação a país, universidade ou, ainda, curso realizado, entre outros, é oportuno enfatizar que iniciativas preventivas para o consumo de drogas psicoativas por estudantes universitários devem ser conduzidas não somente por políticas adotadas em nível nacional, mas devem se somar a elas medidas locais, inclusive dentro da própria universidade, cuja função vai além de formar pessoas para o exercício profissional, mas também para participar ativamente da identificação, do estudo e da solução de problemas sociais, além buscar recursos para a melhoria da qualidade de vida do ser humano.

Nesse sentido, uma vertente de prevenção que se mostrou bastante expressiva neste estudo e que poderia ser estimulada no ambiente universitário foi o aspecto religioso/ espiritual do aluno, tendo em vista que a presença de bem-estar espiritual negativo esteve associada a maior chance de uso abusivo de álcool pelo estudante (OR: 3,325; IC95\%: 1,059-10,441; $p=0,040$ ).

Estudo conduzido pelo Departamento de Enfermagem da Faculdade de Saúde de Gwangyang na Corea ${ }^{26}$, com o objetivo de desenvolver um Programa de Educação para o Cuidado Espiritual voltado para os estudantes de Enfermagem, concluiu que, ao observar dois grupos de sujeitos (experimental: $n=42$ e controle: $n=39$ ), o grupo experimental teve maior pontuação média para a espiritualidade e bem-estar espiritual, comprovando a efetividade do programa, o que, certamente, abre margem para testes em outras universidades.

Outra característica que mostrou ter influência sobre o consumo de drogas pelos estudantes foi a prática religiosa frequente (mais de uma vez por mês), pois a ausência dela esteve associada a maior chance do uso abusivo de álcool pelos estudantes (OR: 3,392; IC95\%: 1,064-10,811; $p=0,039$ ). Da mesma forma, estudo ${ }^{27}$ que analisou a associação entre religiosidade e exposição ao consumo de bebidas alcoólicas evidenciou que o adolescente praticante de atividades religiosas teve menor chance de exposição ao consumo de bebidas alcoólicas (razão de chances OR: 0,71; IC95\%: 0,60$0,83)$. Por conseguinte, é cada vez maior o número de trabalhos que apontam a espiritualidade/religiosidade como importante fator de prevenção ao consumo inicial de drogas por jovens ${ }^{28}$.
De maneira ampla, estudos que avaliam o efeito da espiritualidade na saúde têm apontado relação positiva com vários aspectos do bem-estar físico e mental| ${ }^{13,29}$, o que leva a concluir que se trata de um fenômeno a ser explorado não só no âmbito pessoal, mas também incluído como foco de atenção na assistência à saúde e na prevenção de doenças.

Nesse contexto, quando, neste estudo, os estudantes foram questionados sobre a relevância da espiritualidade na formação do profissional de saúde, 90\% deles afirmaram ser importante (Tabela 2). Essa questão remete à necessidade percebida pelo estudante de apreender o cuidado além da dimensão física, de desenvolver a capacidade de se tornar sensível quanto à dimensão espiritual do paciente, proporcionando assistência holística que resulte em melhor qualidade de vida e de bem-estar.

Também, em estudo ${ }^{30}$ que comparou os conhecimentos e atitudes dos docentes e discentes de Enfermagem quanto à interface espiritualidade, religiosidade e saúde, foi possível identificar que $77 \%$ dos sujeitos sentiam vontade de abordar o assunto; entretanto, somente $36 \%$ julgavam-se preparados e a maioria acreditava que a universidade não proporcionava todas as informações necessárias sobre o tema.

Seguramente, a inclusão da espiritualidade nas práticas de saúde e mesmo na formação profissional, ou ainda como recurso para o desenvolvimento de hábitos de vida saudáveis, é um grande desafio, haja vista a subjetividade do fenômeno e até mesmo o estigma de pessoalidade que o envolve. Particularmente, para a Enfermagem, a mudança de paradigma relacionado à espiritualidade está ligada à falta de formação dos enfermeiros e dos professores para a dimensão espiritual, porém é necessário que haja preparação dos estudantes para a avaliação de necessidades espirituais e para a prestação dos cuidados espirituais ${ }^{31}$, uma vez que a enfermagem é a profissão responsável pela assistência integral ao indivíduo, seja no âmbito físico, social, psicológico ou espiritual.

O conhecimento sobre a espiritualidade pelo futuro profissional cumula também em benefícios pessoais, visto que a espiritualidade permeia questões que resultam em senso de paz interior e de bem-estar ${ }^{32}$. Por sua vez, estes podem ser alcançados com práticas que vão além de limites culturais ou religiosos, ou seja, métodos de meditação, contemplação, oração vocal ou mental, imagens orientadas, musicoterapia, entre outros, que são atividades de cunho espiritual.

Certamente, as discussões sobre o tema não se encerram. Por se tratar de um estudo seccional, não se pode generalizar os resultados e também não é possível avaliar se as associações encontradas são causais nem avaliar a direção da causalidade. No entanto, estudos como ora apresentados permitem não só contribuir com evidências científicas sobre a realidade vivenciada no ambiente universitário, mas também assinalar mecanismos de proteção, para que seja possível construir ações de orientação para a prevenção, para a 
promoção da saúde e para a redução dos riscos e dos danos associados ao consumo de álcool e outras drogas.

Futuros estudos precisam ser conduzidos a fim de estabelecer os mecanismos moduladores do uso de álcool e outras drogas e como eles podem ser empregados para uma população tão vulnerável como a dos estudantes, a fim de fornecer subsídios para a implantação de medidas que garantirão melhor qualidade em sua formação profissional.

\section{CONCLUSÃO}

Este estudo revelou que a espiritualidade pode influenciar positivamente no uso de substâncias psicoativas entre estudantes universitários, uma vez que escores negativos de bem-estar espiritual e ausência de prática religiosa frequente estiveram associados a maior uso abusivo, especialmente de álcool, considerado uma droga socialmente aceita.

Dessa forma, iniciativas preventivas ao consumo de drogas psicoativas, vinculadas à prática de atividades espirituais, devem ser conduzidas em nível individual e comunitário, inclusive na universidade, de forma que os estudantes possam utilizar a própria espiritualidade como estratégia para a manutenção da saúde, para que posteriormente possam usar esse recurso em sua atuação profissional.

\section{CONTRIBUIÇÕES INDIVIDUAIS}

Roberta de Paiva Silva - Concepção e desenho do estudo, análise e interpretação dos dados, elaboração do artigo e aprovação da versão final para publicação.

Priscila de Souza - Concepção e desenho do estudo, análise e interpretação dos dados, elaboração do artigo e aprovação da versão final para publicação.

Denismar Alves Nogueira - Análise e interpretação dos dados, revisão crítica relevante do conteúdo intelectual e aprovação final da versão a ser publicada.

Denis da Silva Moreira - Concepção e desenho do estudo, revisão crítica relevante do conteúdo intelectual e aprovação final da versão a ser publicada.

Erika de Cássia Lopes Chaves - Concepção e desenho do estudo, análise e interpretação dos dados, redação do artigo, revisão crítica relevante do conteúdo intelectual e aprovação final da versão a ser publicada.

\section{CONFLITOS DE INTERESSE}

Os autores declaram não ter nenhum conflito de interesses relativo ao presente artigo. $O$ projeto de pesquisa foi financiado pelo Conselho Nacional de Desenvolvimento Científco e Tecnológico (CNPq).

\section{AGRADECIMENTOS}

Ao Conselho Nacional de Desenvolvimento Científico e Tecnológico (CNPq), pelo provimento de bolsa de iniciação científica.

\section{REFERÊNCIAS}

1. Pillon SC, Webster CMC. Teste de identificação de problemas relacionados ao uso de álcool entre estudantes universitários. Rev Enferm UERJ. 2006;14(3):325-32.

2. Marques ACPR, Cruz MS. 0 adolescente e 0 uso de drogas. Rev Bras Psiquiatr. 2010;22(Supl II):32-6.

3. Brasil. Presidência da República. Secretaria Nacional de Políticas sobre Drogas. I Levantamento Nacional sobre o Uso de Álcool, Tabaco e Outras Drogas entre Universitários das 27 Capitais Brasileiras/Secretaria Nacional de Políticas sobre Drogas; GREA/IPQ-HCFMUSP; organizadores Arthur Guerra de Andrade, Paulina do Carmo Arruda Vieira Duarte, Lúcio Garcia de Oliveira. Brasília: SENAD; 2010.

4. Santos EO, Santos-Oliveira MFS, Kauark FS, Manhães FC. Abordagem sobre a prevenção das drogas no contexto escolar. Inter Science Place. 2011;4(17):18-40.

5. Rodrigues AP, Oliveira AS, Zaleski EGF, Arantes SL. Avaliação do nível de propensão para 0 desenvolvimento do alcoolismo entre estudantes do curso de graduação em enfermagem da Universidade Católica Dom Bosco. SMAD Rev Eletrônica Saúde Mental Álcool Drog. 2007;3(1)

6. Santos JL, Bueno SMV. Educação para a morte a docentes e discentes de enfermagem: revisão documental da literatura científica. Rev Esc Enferm USP. 2011;45(1):272-6.

7. Sanchez ZDM, Nappo SA. A religiosidade, a espiritualidade e o consumo de drogas. Rev Psiq Clín. 2007;34(1):73-81.

8. Abdala AG, Rodrigues WG, Torres A, Rios MC, Brasil MS. A religiosidade/espiritualidade como influência positiva na abstinência, redução e/ou abandono do uso de drogas. Form Vivências Estud (Impr). 2009;2(3):447-60

9. Mohr WK. Spiritual issues in psychiatric care. Perspect Psychiatr Care. 2006;42(3):74-183.

10. Panzini RG, Rocha NS, Bandeira DR, Fleck MPA. Qualidade de vida e espiritualidade. Rev Psiq Clín. 2007;34(3):105-15.

11. Savioli RM. Fronteiras da ciência e da fé. São Paulo: Gaia; 2006. p. 175.

12. Volcan SMA, Sousa PLR, Mari JJ, Horta BL. Relação entre bem-estar espiritual e transtornos psiquiátricos menores: um estudo transversal. Rev Saúde Pública. 2003;37(4):440-5.

13. Marques LF. A saúde e 0 bem-estar espiritual em adultos porto-alegrenses. Psicol Ciênc Prof. 2003;23(2):56-65.

14. Ellison G. Spiritual well-being: conceptualization and measurement. J Psychol Theology. 1983;11(4):330-40

15. Henrique IFS, Micheli D, Lacerda RB, Lacerda LA, Formigoni MLOS. Validação da versão brasileira do teste de triagem do envolvimento com álcool, cigarro e outras substâncias (ASSIST). Rev Assoc Med Bras. 2004;50(2):199-206.

16. Funai A. 0 uso do álcool e religiosidade em estudantes de enfermagem [tese]. Ribeirão Preto: Escola de Enfermagem de Ribeirão Preto da Universidade de São Paulo; 2010.

17. WHO ASSIST Working Group. The alcohol, Smoking and substance Involvement Screening Test (ASSIST): development, reliability and feasibility. Addiction. 2002;97(9):1183-94.

18. Paloutzian $R$, Elisson C. Loneliness, spiritual well-being and the quality of life. Wiley. 1982;224-37.

19. Gastaud MB, Souza LDM, Braga L. Bem-estar espiritual e transtornos psiquiátricos menores em estudantes de Psicologia: estudo transversal. Rev Psiquiatr Rio Gd Sul. 2006;28(1):12-8

20. Chiapetti N, Serbena CA. Uso de álcool, tabaco e drogas por estudantes da área da saúde de uma universidade de Curitiba. Psicol Reflex Crit. 2007;20(2):303-13.

21. Botti NCL, Lima AFD, Simões WMB. Uso de substâncias psicoativas entre acadêmicos de enfermagem da Universidade Católica de Minas Gerais. SMAD Rev Eletrônica Saúde Mental Álcool Drog. 2010;6(1):1-6.

22. Picoloto TCG, Libardoni LFC, Migott AMB, Geib LTC. Prevalência e fatores associados com 0 consumo de substâncias psicoativas por acadêmicos de enfermagem da Universidade de Passo Fundo. Ciênc Saúde Coletiva. 2010;15(3):645-54. 
23. Leiva Diaz V, Vasters GP, Costa Junior ML. Caracterización de estudiantes de la carrera de enfermeira sobre consumo de drogas lícitas e ilícitas. Rev Latino-Am Enfermagem. 2010;18(Spe):565-72

24. México. Consejo Nacional Contra las Adicciones - Secretaría de Salud. Encuesta Nacional de Adicciones. Cuernavaca: Instituto Nacional de Salud Pública; 2008.

25. Hanson G, Venturelli PJ. Drugs and society. 6th ed. Unites States of America: Jones and Barlett Publishers Inc; 2001.

26. Chung MJ, Eun Y. Development and effectiveness of a spiritual care education program for nursing students--based on the ASSET model. J Korean Acad Nurs. 2011;41(5):673-83.

27. Bezerra J, Barros MVG, Tenório MCM, Tassitano RM, Barros SSH, Hallal PC. Religiosidade, consumo de bebidas alcoólicas e tabagismo em adolescentes. Rev Panam Salud Publica. 2009;26(5):440-6
28. Pillon SC, Santos MA, Gonçalves AMS, Araújo KM, Funai A. Fatores de risco, níveis de espiritualidade e uso de álcool em estudantes de dois cursos de enfermagem. SMAD Rev Eletrônica Saúde Mental Álcool Drog. 2010;6(spe):493-513.

29. Guimarães, HP, Avezum, A. 0 impacto da espiritualidade na saúde física. Rev Psiq Clín. 2007;34(1):88-94.

30. Tomasso CS, Beltrame IL, Lucchetti G. Knowledge and attitudes of nursing professors and students concerning the interface between spirituality, religiosity and health. Rev LatinoAm Enfermagem. 2011;19(5):1205-13.

31. Caldeira S. A espiritualidade no currículo de enfermagem. Rev Nursing Portuguesa. 2012;282:18-21.

32. Swinton J, Pattison S. Moving beyond clarity: towards a thin, vague, and useful understanding of spirituality in nursing care. Nurs Philos. 2010;11(4):226-37. 